\title{
Strategic Faults in Implementation of Hospital Accreditation Programs in Developing Countries: Reflections on the Iranian Experience
}

\author{
Aidin Aryankhesal ${ }^{12^{*}}$
}

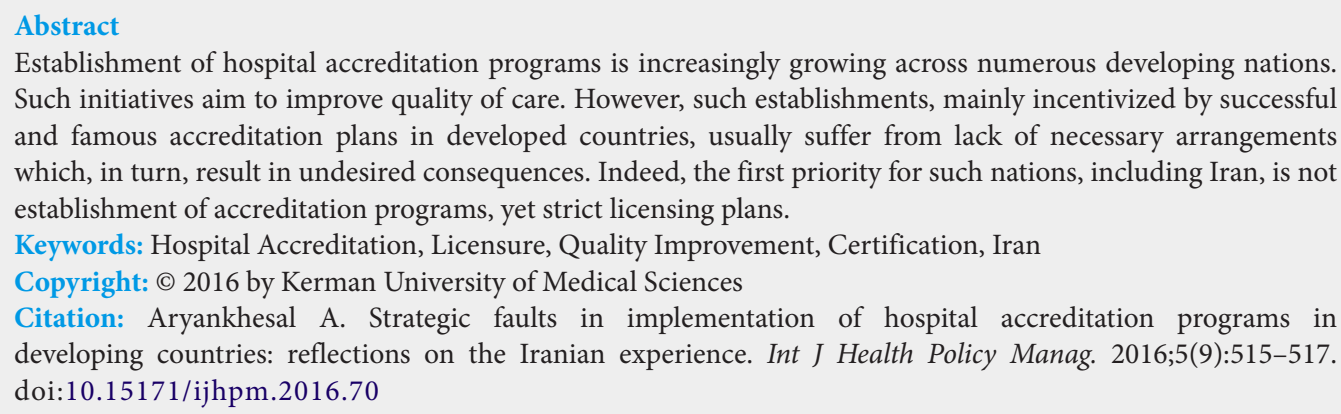

Establishment of hospital accreditation programs is increasingly growing across numerous developing nations. Such initiatives aim to improve quality of care. However, such establishments, mainly incentivized by successful and famous accreditation plans in developed countries, usually suffer from lack of necessary arrangements which, in turn, result in undesired consequences. Indeed, the first priority for such nations, including Iran, is not establishment of accreditation programs, yet strict licensing plans.

Keywords: Hospital Accreditation, Licensure, Quality Improvement, Certification, Iran Copyright: @ 2016 by Kerman University of Medical Sciences

Citation: Aryankhesal A. Strategic faults in implementation of hospital accreditation programs in developing countries: reflections on the Iranian experience. Int J Health Policy Manag. 2016;5(9):515-517. doi:10.15171/ijhpm.2016.70

\section{Article History:}

Received: 18 April 2016 Accepted: 30 May 2016 ePublished: 1 June 2016

View Video Summary

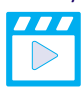

*Correspondence to:

Aidin Aryankhesal

Email: aryankhesal.a@iums.ac.ir

\section{Similarities Between Accreditation and Olympic Games} Hospital accreditation programs, with a history of more than six decades in developed nations, ${ }^{1}$ may have some similarities with the Olympic Games. Both aimed to improve the quality and outcomes, one in care and services and the other in physical abilities and records. Both work through establishment of some type of competition, the former among hospitals and the latter among athletes and nations. Indeed, the participants may first pass some basic requirements, the basic level of standards or the qualification records to be qualified for the competition. In both events, the participants are usually among the fittest ones, well-organized hospitals and the nationally selected athletes, respectively. Therefore, the expected quality of participants in the events is much higher than what is expected from the average of typical hospitals or athletes. In both, the winners are rewarded, by a gold seal, or gold medal, which brings them other achievements such as reputation and possibly financial benefits. Both events need funding, which usually come from application fees paid by applicant hospitals or the membership fees, paid by member nations. Finally, both events take place every three-four years. Such similarities are seen between most accreditation programs of developed nations and the Olympic Games. However, the story is rather different in some developing countries. ${ }^{2-5}$

\section{Different Story in Iran and Some Developing Countries} Developing countries historically suffer from a lack of effective control over hospital establishment considerations $s^{6,7}$ and Iran is no exception. Such considerations may include need assessment, site selection, mapping, construction and material, wards and spaces, staffing, and equipping. Finally after the establishment, hospitals should be monitored for their clinical processes and outcomes.
Experiencing patient dissatisfaction and undesirable status of hospitals, especially after the years of 1980-1988 Iran-Iraq war, ${ }^{8}$ Iran's government decided to establish a program to solve the problems. What established was a national hospital grading or accreditation program in 1998. ${ }^{9}$ Indeed, the accreditation program implemented was mainly influenced by the reputation and impressive impacts of accreditation programs in developed nations such as the United States. However, Government's expectations from the accreditation program to overhaul all hospitals led to some unusual and odd legislations and mechanisms within the accreditation program, even in its new version, launched in 2012.

The first different policy from what happens in the United States, is Iran's compulsory accreditation program. Iran's Ministry of Health and Medical Education (MoHME), as both the accreditation body and agent, obliged all hospitals, ${ }^{10}$ not voluntarily as happens in most accreditation programs, ${ }^{11}$ to prepare themselves for the accreditation. Therefore, there would be no minimum entrance requirement for accreditation. Such obligation from the government side for national accreditation was probably in order to make sure that hospitals would not miss basic requirements, as there was no other effective means that check and control the basics across hospitals. Such basic requirements could be related to space and building such as a separate entrance for emergency department (ED), minimum of $9 \mathrm{~m}^{2}$ space per bed in any inpatient room, etc ${ }^{12}$ or human resources that hospitals have no or less control over them. ${ }^{13}$ Against this kind of standards, hospitals may resist or game because they could not make necessary changes easily. ${ }^{13,14}$ Moreover, we know that the effectiveness of Iranian hospital accreditation comes from its pay for performance (P4P) policy, according to which the audit scores would define hospitals' level of charges. ${ }^{15}$ Such $\mathrm{P} 4 \mathrm{P}$ policy conflicts with the MoHME's interests; MoHME 
owns most of Iran's hospitals and strict audits would decrease hospitals' revenue that would be a huge financial burden on the government. Consequently, the MoHME's role as both accreditation agent and owner of hospitals results in easygoing policies through auditing public hospitals. ${ }^{16}$ The consequences would be even worse regarding that various basic standards of hospital had been neglected when they were licensed. ${ }^{17}$ Therefore, the government's compulsory auditing policy would not work due to basic shortcomings in hospitals and conflict of interest for the government.

The second difference between Iran's hospital accreditation program and the USA's was its level of standards. Contrary to most accreditation programs, ${ }^{18}$ the Iranian accreditation standards were not and could be optimum or even at a high level. ${ }^{15}$ Therefore, what is audited through the accreditation would be a long list of standards including a mix of basic ones and probably some advanced. Indeed, the audited standards would be diluted in favor of low and basic ones and should be run every year because there is no other effective controlling device over hospitals' standards. The long list of requirements, which includes 37 domains with more than 1000 measures, ${ }^{12}$ forces hospitals staff to carry out heaps of paperwork annually, not once in three-four years, and leads to their frustration. ${ }^{14}$ Another mismatch in our comparison between the USA's hospital accreditation program and Iran's relates to the funding considerations. Owing to that hospital accreditation is a mandatory program in Iran, the governments cannot charge hospitals for application, which otherwise would seem irrational. Therefore, all expenses of running the accreditation are covered by the public budget. The volume of expenses is highly enormous considering that all hospitals, about 900, should be audited at least once a year. Hence the sustainability of such programs will be questionable. ${ }^{19}$ Therefore, owing to the high costs of running the accreditation program and its time consuming process, the MoHME would have less or no budget and time for development and revision plans over the program. As a result, the audited standards are usually out of date or invalid. ${ }^{14}$

\section{Strategic Faults}

Considering the above mentioned situations, one may suggest that some developing nations, have made a strategic fault in the establishment of hospital accreditation plans. In Pakistan, for example, the accreditation standards could not fit all hospitals because some were poor even in basic requirements. ${ }^{20}$ In Lebanon, also some shortcomings like staffing and lack of quality improvement culture in some hospitals were reported as challenges of establishment of accreditation programs which should be solved before the establishment. ${ }^{21}$ In Iran as well, the accreditation programs can work well as quality improvement driver if basic standards and requirements are passed in hospitals beforehand. Otherwise, the accreditation program should include lots of basic domains and indicators which will be frustrating. ${ }^{15,22}$ The basic standards and requirements, such as building and structure measures, can be checked by authorities once at first. In the United States, the Department of Health and Center for Medicare and Medicaid Services (CMS) have rigid regulations for licensing hospitals at their establishment point and monitor the basic regulations and standards annually. ${ }^{23}$ Therefore, hospitals start working only when they are truly licensed. Among the licensed hospitals, those seeking reputation and larger share of the market, should work on the advanced quality measures defined by a third party non-governmental agency such as the Joint Commission (TJC). Indeed what saves developed nations' hospitals from basic shortcomings are not accreditation programs, but the initial licenses granted to hospitals by the governments through the Ministry/Department of Health or other public authorities. The accreditation programs across them usually ensure continuous improvement of quality. In the current situation, what probably will work for developing nations such as Iran, for improvement of hospitals, can be a model demonstrated in the Figure. First the nations need a strong institute that its technical job is licensing. Such institution should not let the hospitals become established with very severe shortcomings, nonstandard setting and spaces for instance, that are impossible or really difficult to change later. Many hospitals in Iran are located in busy and heavy-traffic avenues that make accessing them very difficult even in emergencies. Some hospital also are licensed when they have no sufficient space for their beds. Nevertheless, these hospitals are accredited, while cannot solve their basic problems.

The next step after establishment of a rigid licensing mechanism, can be establishment of certification mechanisms. The certification mechanisms would be voluntary audits which focus on one or a few aspects of volunteer hospitals' performance. Examples of such certificates may include "patient safety friendly" or "baby friendly" certificates for hospitals apply and follow the necessary standards. The certificate can improve hospitals' reputation and fame so increase their share of the market among their rivals. This kind of initiatives can make healthcare organizations and their culture mature enough for the establishment of accreditation and continuous quality improvement programs. ${ }^{24}$ Hence, a gradual process for establishment of accreditation programs in Iran or developing nations may follow the steps shown in the Figure.

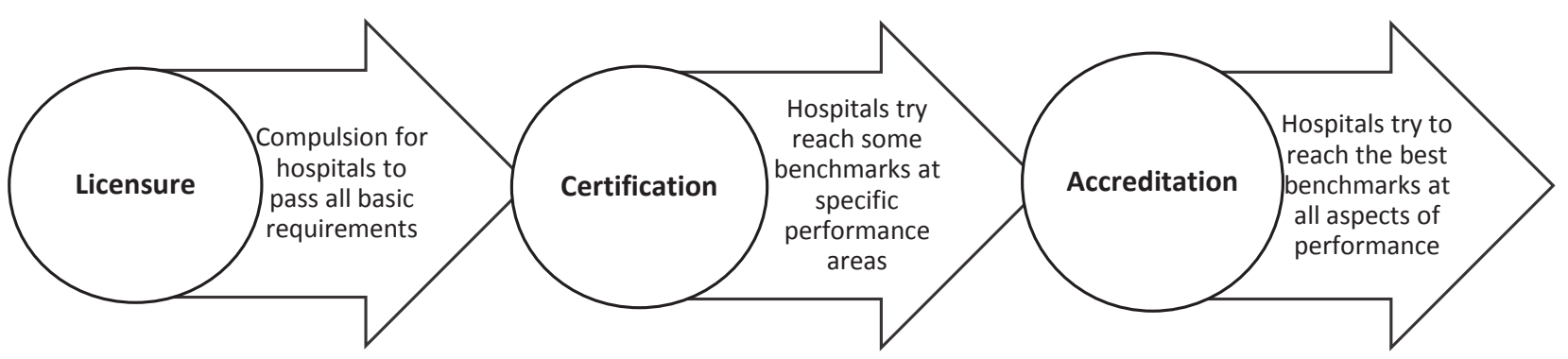

Figure. Suggested Steps for the Establishment of Accreditation Programs for Healthcare Organizations in Developing Countries. 


\section{Conclusion}

Accreditation systems in the majority of developing countries, although might have positive impacts on quality of care, ${ }^{25,26}$ are possibly established without considering all necessary arrangements. Strict rules for licensing the newly established hospitals and useful benchmarks for certification plans are among neglected necessary arrangements. Such accreditation programs, not only have no revenue for the accreditation agents, eg, the government in Iran, still impose a great burden of cost on developing countries. ${ }^{27}$ The dysfunctional consequences may even question the trade-off between the benefits and harms of the accreditation programs. ${ }^{14}$ Indeed policy-makers in developing nations should regard establishment of accreditation systems carefully and as "beginners," ${ }^{28}$ after establishment of necessary infrastructures for healthcare organizations.

Ethical issues

Not applicable.

Competing interests

Author declares that he has no competing interests.

Author's contribution

AA is the single author of the paper.

References

1. Patterson $\mathrm{CH}$. Joint Commission on accreditation of healthcare organizations. Infect Control Hosp Epidemiol. 1995;16(1):36-42. doi:10.1086/647001

2. Novaes HM. Hospital accreditation in developing countries. In: Al-Assaf AF, ed. Health Care Quality: An International Perspective. New Delhi: WHO; 2001:241. http://apps.searo. who.int/PDS_DOCS/B0110.pdf\#page $=245$.

3. El-Jardali F, Jamal D, Dimassi H, Ammar W, Tchaghchaghian V. The impact of hospital accreditation on quality of care: perception of Lebanese nurses. Int J Qual Health Care. 2008;20(5):363371. doi:10.1093/intqhc/mzn023

4. Galukande M, Katamba A, Nakasujja N, et al. Developing hospital accreditation standards in Uganda. Int $\mathrm{J}$ Health Plan Manage. 2015. doi:10.1002/hpm.2317

5. Bateganya M, Hagopian A, Tavrow P, Luboga S, Barnhart S. Incentives and barriers to implementing national hospital standards in Uganda. Int J Qual Health Care. 2009;21(6):421426. doi:10.1093/intqhc/mzp044

6. Wilson R, Michel P, Olsen S, et al. Patient safety in developing countries: retrospective estimation of scale and nature of harm to patients in hospital. BMJ. 2012;344:e832. doi:10.1136/bmj. e832

7. Harrison R, Cohen AW, Walton M. Patient safety and quality of care in developing countries in Southeast Asia: a systematic literature review. Int J Qual Health Care. 2015. doi:10.1093/ intqhc/mzv041

8. Hajizadeh M, Nghiem HS. Hospital care in Iran: an examination of national health system performance. International Journal of Healthcare Management. 2013;6(3):201-210. doi:10.1179/2047 971913y.0000000042

9. Hashjin AA, Kringos DS, Manoochehri J, Aryankhesal A, Klazinga NS. Development and impact of the Iranian hospital performance measurement program. BMC Health Serv Res. 2014;14:448. doi:10.1186/1472-6963-14-448

10. Aryankhesal A, Sheldon T. Effect of the Iranian hospital grading system on patients' and general practitioners' behaviour: an examination of awareness, belief and choice. Health Serv Manage Res. 2010;23(3):139-144. doi:10.1258/hsmr.2009.009028

11. Alkhenizan $A$, Shaw $C$. Impact of accreditation on the quality of healthcare services: a systematic review of the literature. Ann Saudi Med. 2011;31(4):407. doi:10.4103/0256-4947.83204
12. Ministry of Health and Medical Education (MoHME). Hospital accreditation standards in Iran. Tehran: MoHME, Deputy of Curative Affairs; 2010.

13. Agrizzi D, Agyemang G, Jaafaripooyan E. Conforming to accreditation in Iranian hospitals. Accounting Forum. 2016. doi:10.1016/j.accfor.2016.02.002

14. Aryankhesal A, Sheldon TA, Mannion R, Mahdipour S. The dysfunctional consequences of a performance measurement system: the case of the Iranian national hospital grading programme. J Health Serv Res Policy. 2015;20(3):138-145. doi:10.1177/1355819615576252

15. Aryankhesal A, Sheldon TA, Mannion R. Role of pay-forperformance in a hospital performance measurement system: a multiple case study in Iran. Health Policy Plan. 2013;28(2):206214. doi:10.1093/heapol/czs055

16. Aryankhesal A, Sheldon TA, Mannion R. Impact of the Iranian hospital grading system on hospitals' adherence to audited standards: an examination of possible mechanisms. Health Policy. 2014;115(2):206-214. doi:10.1016/j.healthpol.2013.11.004

17. Aghaei Hashjin A, Delgoshaei B, Kringos DS, Tabibi SJ, Manouchehri J, Klazinga NS. Implementing hospital quality assurance policies in Iran: Balancing licensing, annual evaluation, inspections and quality management systems. Int J Health Care Qual Assur. 2015;28(4):343-355. doi:10.1108/ ijhcqa-03-2014-0034

18. Valentine B. Licensing, accreditation and quality improvement. Australian Journal of Public Administration. 2007;66(2):238-247. doi:10.1111/j.1467-8500.2007.00531.x

19. Bukonda $\mathrm{N}$, Tavrow $\mathrm{P}$, Abdallah $\mathrm{H}$, Hoffner $\mathrm{K}$, Tembo J. Implementing a national hospital accreditation program: the Zambian experience. Int J Qual Health Care. 2002;14(Suppl 1):7-16. doi:10.1093/intqhc/14.suppl_1.7

20. Sax S, Marx M. Local perceptions on factors influencing the introduction of international healthcare accreditation in Pakistan. Health Policy Plan. 2014;29(8):1021-1030. doi:10.1093/heapol/ czt084

21. El-Jardali F, Ammar W, Hemadeh R, Jamal D, Jaafar M. Improving primary healthcare through accreditation: baseline assessment of readiness and challenges in lebanese context. Int J health Plan Manage. 2013;28(4):e256-e279. doi:10.1002/ hpm.2170

22. Bahadori M, Ravangard R, Alimohammadzadeh K. The accreditation of hospitals in iran. Iran $J$ Public Health. 2015;44(2):295-296. doi:10.1136/bmj.h4407

23. Centers for Medicare \& Medicaid Services. Quality inititives General information. https://www.cms.gov/medicare/quality-initiatives-patient-assessment-instruments/qualityinitiativesgeninfo/ index.html. Accessed May 28, 2016. Published 2016.

24. Davies HT, Nutley SM, Mannion R. Organisational culture and quality of health care. Qual Health Care. 2000;9(2):111-119. doi:10.1136/qhc.9.2.111

25. Salmon JW, Heavens J, Lombard C, Tavrow P. The impact of accreditation on the quality of hospital care: KwaZulu-Natal province Republic of South Africa. Washington, DC: US Agency for International Development, Quality Assurance Project; 2003.

26. Jafary $\mathrm{FH}$, Ahmed $\mathrm{H}$, Kiani J. Outcomes of primary percutaneous coronary intervention at a joint commission international accredited hospital in a developing country--can good results, possibly similar to the west, be achieved? J Invasive Cardiol. 2007;19(10):417-423.

27. Smits H, Supachutikul A, Mate KS. Hospital accreditation: lessons from low-and middle-income countries. Globalization and health. 2014;10(1):1. doi:10.1186/s12992-014-0065-9

28. Saleh SS, Alameddine MS, Natafgi NM. Beyond accreditation: a multi-track quality-enhancing strategy for primary health care in low-and middle-income countries. Int $\mathrm{J}$ Health Serv. 2014;44(2):355-372. doi:10.2190/hs.44.2.k 